\title{
Sextant
}

Revue de recherche interdisciplinaire sur le genre et la sexualité

$37 \mid 2020$

Le rideau déchiré

\section{Psychanalyse pendant la guerre froide}

Politiques sexuelles et christianisation de la « science juive »

Dagmar Herzog

\section{(2) OpenEdition \\ 12 Journals}

Édition électronique

URL : https://journals.openedition.org/sextant/327

DOI : $10.4000 /$ sextant.327

ISSN : 2795-8736

Éditeur

Éditions de l'Université de Bruxelles

\section{Édition imprimée}

Date de publication : 1 décembre 2020

Pagination : 41-52

ISBN : 978-2-8004-1744-8

ISSN : $1370-267 X$

\section{Référence électronique}

Dagmar Herzog, «Psychanalyse pendant la guerre froide », Sextant [En ligne], 37 | 2020, mis en ligne le 01 novembre 2021, consulté le 25 janvier 2022. URL : http://journals.openedition.org/sextant/327 ;

DOI : https://doi.org/10.4000/sextant.327

\section{(c) (i) (2)}

La revue Sextant est mise à disposition selon les termes de la Licence Creative Commons Attribution Pas d'Utilisation Commerciale - Partage dans les Mêmes Conditions 4.0 International. 


\title{
Psychanalyse pendant la guerre froide Politiques sexuelles et christianisation de la « science juive »
}

\author{
Dagmar HeRzoG
}

Que se passe-t-il quand un corpus conceptuel, né dans l'Europe centrale fin de siècle, traverse l'Atlantique et arrive aux États-Unis, plusieurs décennies plus tard, pendant une période d'intense anticommunisme ? Quelles conséquences eurent, à long terme, l'association que firent entre la judéité et un (sur)intérêt pour la sexualité les détracteurs de la psychanalyse, qu'ils aient été nazis ou chrétiens ? Autre question : en quoi l'évolution historique du début de la guerre froide aux États-Unis permetelle d'expliquer à la fois le succès initial remarquable qu'y a connu le freudisme et la réputation douteuse - voire souvent absolument déplorable - qu'il a aujourd'hui, aussi bien auprès des psychiatres que de l'opinion publique ?

En nul autre lieu et à nulle autre époque, la psychanalyse freudienne n'a connu pareil succès, et la psychiatrie n'a été aussi psychanalytique que dans les deux premières décennies de la guerre froide aux États-Unis, entre 1949 et 1969. Ce fut l'« âge d'or » de la psychanalyse, l'époque et le lieu où elle gagna le plus de terrain, que ce soit dans la culture médicale ou dans la culture populaire. Ce fut aussi un moment et un lieu où la psychanalyse se montra extrêmement conservatrice - incontestablement misogyne, homophobe et autoritariste. Deux décennies plus tard, son autorité culturelle et médicale s'effondrerait, presque complètement, sous les coups des militants pour les droits des femmes et des homosexuel-les, des pacifistes de la New Left et, en même temps, en raison de l'emprise, renouvelée, de la psychiatrie biologique et de la diffusion du « développement personnel » par les médias (à un moment pourtant où la psychanalyse était de mieux en mieux vue en Europe de l'Ouest et en Amérique latine, portée précisément par ces mêmes mouvements de défense des droits des femmes et des homosexuel-les et cette même New Left qui étaient en train d'anéantir le freudisme aux États-Unis). 
Il ne fait aucun mystère que la psychanalyse américaine était conservatrice - que ce soit par simple refus de l'engagement ou en renforçant des valeurs conformistes. On explique généralement ce phénomène par le fait que la psychanalyse s'était adaptée à une culture américaine qui valorisait l'optimisme et le développement personnel. Les historiens proposent, en outre, quatre ou cinq autres explications : 1) l'effet émollient de la médicalisation (puisque, aux États-Unis, jusqu'aux années 1980, seuls les médecins avaient le droit de pratiquer comme analystes), 2) le fait que l'on ait fait taire ou que l'on ait expulsé les analystes les plus radicaux, dans ce qu'on pourrait appeler une épuration interne de la profession, 3) une pression de l'État, qui prit la forme d'une lutte anticommuniste et d'une surveillance intimidante - où les émigrés aux accents étrangers, pour nombre d'entre eux d'anciens sociaux-démocrates, firent l'objet d'une suspicion toute particulière ${ }^{1}$. (Une variante récente de cette hypothèse impute la responsabilité à d'anciens membres du Parti communiste des États-Unis d'Amérique, nés aux États-Unis, qui auraient pratiqué une forme d'autocensure tout en restant, au fond, staliniens ${ }^{2}$.) Enfin, l'explication 4) - celle qui prévaut actuellement - suggère que la psychanalyse doit être comprise elle-même comme une " survivante de la Shoah »-qui, traumatisée, tenta désespérément de se fondre dans la société d'accueil. Les États-Unis avaient, de fait, sauvé en les accueillant les psychanalystes et leurs familles qui fuyaient les persécutions nazies ${ }^{3}$. De toute évidence, ces psychanalystes avaient de nombreuses et de pressantes raisons de se montrer normatifs dans leurs thérapies ou de faire preuve de prudence en ne proposant, comme conseils, qu'une bouillie insipide.

Cette dernière explication, en particulier, surestime cependant l'influence des quelque 190 analystes juifs qui avaient fui le nazisme. Et aucune de ces explications ne parvient à justifier l'indéniable conservatisme en matière de sexualité de la psychanalyse américaine. Pour ce faire, je souhaiterais avancer plusieurs arguments. Tout d'abord, et c'est là le premier moment de mon argumentation, j'estime que nous devons considérer que la « science juive » qu'aurait été la psychanalyse, et avec elle la spécialité médicale de la psychiatrie, a été soumise à un processus qu'on ne peut désigner autrement que par le terme de «christianisation » dans les États-Unis de l'après-guerre - à la réserve près qu'à cette époque, la religion, et le christianisme en particulier (surtout dans ses composantes favorables au capitalisme, proches des milieux des affaires et patriotiques), se « psychologisait ${ }^{4}$ aussi de plus en plus. Mon

${ }^{1}$ E. A. Danto, « Have You No Shame? - American Redbaiting of Europe's Psychoanalysts », in J. Damousi et M. Ben Plotkin (dir.), Psychoanalysis and Politics: Histories of Psychoanalysis under Conditions of Restricted Political Freedom, New York, Oxford University Press, 2012, p. 213-231.

${ }^{2}$ Arnold Richards, communication privée avec l'auteure.

${ }^{3}$ E. Kuriloff, "Revelations in Psychoanalytic History », Psychoanalytic History, Contemporary Psychoanalysis, 45/4, 2009, p. 577-580 ; L. Aron et K. STARr, A Psychotherapy for the People: Toward a Progressive Psychoanalysis, New York/Londres, Routledge, 2013.

${ }^{4}$ Le texte de référence est le suivant: W. Herberg, Protestant-Catholic-Jew: An Essay in American Religious Sociology, New York, Anchor Books, $2^{\mathrm{e}}$ éd., 1960. Édition française : Protestants, catholiques et israélites, la religion dans la société aux États-Unis, essai de 
deuxième argument montre que les débats sur la place de la libido dans l'entreprise freudienne et postfreudienne sont absolument essentiels pour comprendre comment la psychanalyse, et avec elle la psychiatrie américaine de l'après-guerre, a évolué comme elle l'a fait. Étaient en jeu à la fois le statut de la libido comme un objet d'étude scientifique, la place qu'elle occupe dans le comportement humain et ce que le terme « libido » était censé signifier. Enfin, mon troisième argument est le suivant : les contours comme le contenu de la psychanalyse dans les États-Unis d'après-guerre ont été structurés par des rivalités dans une série d'affrontements successifs. Ils eurent lieu d'abord avec ceux qu'on appelle les « néofreudiens » comme Franz Alexander, Harry Stack Sullivan, Erich Fromm et surtout Karen Horney - dont le féminisme exaspérait ses adversaires tandis que sa popularité les faisait enrager - ; puis avec des responsables religieux, surtout catholiques ; enfin, alors que ces deux premières confrontations semblaient avoir été gérées efficacement, avec le sexologue Alfred Kinsey.

$\mathrm{Au}$ cœur de ces trois rivalités se trouvait une profonde incertitude quant à la place centrale donnée à la libido dans l'édifice freudien. Cette incertitude était, naturellement, à la fois logique (après tout, il est vrai que les êtres humains ont d'autres motivations) et problématique. De plus, l'agressive rhétorique nazie avait contribué à aggraver la gêne face à la centralité de la libido. Le discours nazi des années 1930 accusait en effet Freud de « fantasmes obscènes » et considérait que la psychanalyse ne contenait « rien d'original » - car donner à entendre le contraire « serait faire trop d'honneur à l'improductivité de la race juive »- et qu'elle «n'était rien d'autre que le viol de la culture occidentale par la nation juive $»^{5}$. Les livres de Freud furent brûlés et ses idées furent, en même temps, dénigrées et pillées. Il en allait cependant autrement dans le contexte américain.

La montée de l'engouement pour la psychanalyse aux États-Unis - dans le grand public, dans la psychiatrie et dans les sciences sociales - se produisit dans la deuxième moitié des années 1930 et au début des années 1940. Cette dynamique était principalement impulsée par un groupe (composé à la fois d'immigrés et d'Américains de naissance, de juifs et de non-juifs) souvent désigné sous le terme vague de «néofreudiens ». Ils se caractérisaient par leur intérêt particulier pour la culture, le milieu, les contraintes sociales dans la vie quotidienne de l'adulte, et pas seulement pour le passé de l'enfance. En outre, au lieu de mettre l'accent sur la recherche du plaisir chez l'enfant en bas âge, ils soulignaient l'importance de l'anxiété et du sentiment d'insécurité existentielle. Harry Stack Sullivan

sociologie religieuse, traduction de J. Yardley et G. Serve, Paris, Spes, 1960. Voir également K. Kruse, One Nation Under God: How Corporate America Invented Christian America, New York, Basic Books, 2015 - de même que N. V. Peale, The Power of Positive Thinking, New York, Prentice-Hall, 1952. Première édition française : Votre pensée peut tout, traduction de S. Lechevrel, Paris, Les Presses de la Cité, 1955.

5 Anon., « Die Rolle des Juden in der Medizin », Deutsche Volksgesundheit aus Blut und Boden, août-septembre 1933, p. 15 ; et H. Hunger, « Jüdische Psychoanalyse und deutsche Seelsorge ", in W. Grundmann (dir.), Germanentum, Judentum und Christentum, vol. 2, Leipzig, G. Wigand, 1943. 
et Erich Fromm en étaient les principaux représentants. Karen Horney - qui était proche et s'inspirait de tous deux - synthétisa leurs idées dans son bestseller de 1937, The Neurotic Personality of Our Time (première publication en français : La Personnalité névrotique de notre temps, traduction de Jean Paris, Paris, L'Arche, 1953) et sa suite, le célèbre New Ways in Psychoanalysis (1939) (première publication en français : Les Voies nouvelles de la psychanalyse, 1951). Horney affirmait ouvertement que la «partialité de Freud à porter attention au principe de plaisir, implicite dans la théorie de la libido », devait être remplacée par une « orientation sociologique » dans laquelle le « besoin de sécurité » dans un environnement inéluctablement accablant était le but prééminent de l'existence humaine $^{6}$. Plus généralement, Horney affirmait que "le concept de libido n'est pas démontré [et que] la validité des données concernant les zones érogènes est fortement sujette à caution ${ }^{7}$.

De nos jours, Horney est généralement avant tout considérée comme une féministe et, pour certains puristes, même pas comme une vraie analyste. Elle reste surtout connue pour sa critique de l'idée de l'«envie du pénis » et sa suggestion que les hommes pourraient bien souffrir d'une « envie d'utérus » face à la capacité génésique de la femme ${ }^{8}$. Je pense, cependant, qu'elle doit aussi être comprise comme une théoricienne de la sexualité (et non seulement du genre) et - surtout - comme quelqu'un qui a rethéorisé, d'une manière intéressante, les relations entre la sexualité et les autres dimensions de l'existence. Comme elle l'écrit dans son livre de 1937 « tout comme "tout ce qui brille n'est pas or", tout ce qui ressemble à de la sexualité n'en est pas ». Le simple fait que quelque chose ait l'air sexuel - voire implique des actes sexuels au propre - ne signifie pas que son objet ou sa fonction soient avant tout sexuels, les actes sexuels pouvant répondre à toutes sortes d'autres objectifs émotionnels. De plus, même dans le cas de problèmes sexuels, Horney repense le sens de la relation de cause à effet. Pour elle, « [1] es problèmes sexuels sont plutôt l'effet que la cause de la structure névrotique de la personnalité $»{ }^{9}$. Fromm, également, dans son œuvre majeure de 1941, Escape from Freedom (en français : La Peur de la liberté, traduction de C. Jansens, Paris, Buchet-Chastel, 1963), adoptant le franc-parler de Horney (et en la citant en notes de bas de page), soutient que Freud s'est trompé sur le sens de l'enchaînement causal. Il écrit ainsi que Freud « a inversé la relation de causalité entre zones érogènes et traits de caractère ».

${ }^{6}$ Et elle ajoute alors - puisqu'elle a consacré un chapitre entier à la réfutation du complexe d'EEdipe - que le récit du mythe d'Édipe ne doit pas être réécrit, mais qu'il doit tout simplement être rejeté. « Le facteur pertinent dans la genèse des névroses n'est alors ni le complexe d'Edipe, ni la quête de plaisir pendant l'enfance, mais bien tous les facteurs néfastes qui font qu'un enfant se sent en détresse et désemparé et lui font voir le monde comme un lieu plein de dangers. »

${ }^{7}$ K. Horney, New Ways in Psychoanalysis, New York, Norton, 1939, p. 9-10, 48, 52. Traduction française : Les Voies nouvelles de la psychanalyse, Paris, L'Arche, 1951.

${ }^{8}$ K. Horney, "The Flight from Womanhood: The Masculinity-Complex in Women, as Viewed by Men and by Women », International Journal of Psycho-Analysis, 7, 1926, p. 331.

${ }^{9}$ K. Horney, New Ways in Psychoanalysis, op. cit., p. 10. 
Horney était très appréciée du grand public tout en jouissant d'une très grande considération dans le domaine des sciences sociales où elle avait des disciples dévoués. De fait, ce sont précisément cette popularité et la durable loyauté de ses disciples qui provoqueront l'animosité de ses adversaires. Cependant, déjà à ce moment-là, et très certainement a posteriori, il était et il est toujours impossible de démêler l'écheveau des causes possibles de l'hostilité dirigée contre elle, entre la jalousie professionnelle, la misogynie et un authentique désaccord théorique ${ }^{10}$. Dès 1941 , des psychanalystes hommes se liguèrent contre Horney pour la décrédibiliser professionnellement et la priver de son prestige, et cela, sans discontinuer jusqu'à sa mort en 1952 - qui signerait l'arrêt de mort du néofreudisme en général. Ce dernier ne tarderait pas à être remplacé par l'avènement des « égopsychologues » autour de l'émigré viennois Heinz Hartmann qui, dans les cercles psychanalytiques, a donné son nom à la «période Hartmann » de l'après-guerre.

Étonnamment, le nom de Karen Horney figurait cependant dans la liste que le magazine Time dressa en 1946 des six « plus grands analystes » des États-Unis. Elle était du reste la seule femme à s'y trouver et le Time expliquait obligeamment que les « horneyéens » avaient une « conscience sociale » et qu'ils « contestaient l'idée de Freud selon laquelle la sexualité était tout ». Les cinq autres analystes de la liste du Time étaient Franz Alexander, l'ancien associé de Horney à Chicago (qui avait pris ses distances avec elle dès 1934); le docteur Gregory Zilboorg et le docteur Lawrence Kubie, « les analystes à la mode de Park Avenue », et « les docteurs William et Karl Menninger de la Clinique Menninger de Topeka ${ }^{11}$. Ces cinq hommes refusaient tous les idées de Horney, voire la contraient avec véhémence, en public comme en privé, soit en s'opposant à elle dès le début (comme Zilboorg), soit en réaction à l'intérêt

${ }^{10}$ Sur la façon dont la mort de Horney a scellé le sort du néofreudisme, voir N. MCLAUGHLIN, « Why Do Schools of Thought Fail? Neo- Freudianism as a Case Study in the Sociology of Knowledge », Journal of the History of the Behavioral Sciences, 34/2, printemps 1998, p. 113134. Deux styles sont apparus pour succéder aux néofreudiens. L'un d'eux se désintéressait ouvertement de la question de la sexualité. Les égopsychologues commencèrent à formuler leurs idées entre la fin des années 1940 et le début des années 1950. Ces idées furent mises en application par Heinz Hartmann et ses coauteurs Ernst Krist et Rudolf Loewenstein, à New York, et par son proche collaborateur, Edward Bribing, à Boston ainsi que par le collègue de Karl Menninger, David Rapaport (d'abord à Topeka, puis à Stockbridge). Pour ces égopsychologues, le monde extérieur qui préoccupait tant les néofreudiens continuait à importer, grandement, dans la mesure où ils mettaient l'accent sur l'aptitude individuelle à s'adapter à la réalité. Pour eux, la tâche principale de la psychanalyse, cependant, était d'assister le patient à moduler- et surtout à neutraliser - ses pulsions sexuelles ou d'agression, et plus encore à créer et à développer les fonctions autonomes du moi. Hartmann n'avait pas cherché à théoriser la sexualité en tant que telle, mais il avait tendance à s'en désintéresser, considérant que d'autres avaient déjà traité le sujet de façon exhaustive. L'autre style qui apparut (caractérisé par le propre travail de Karl Menninger, mais qu'on retrouve aussi chez des analystes d'importance nationale comme Robert Knight, Phyllis Greenacre, Leo Rangel ou encore Ralph Greenson, parmi tant d'autres) insistait sur l'importance des questions liées à la libido et rétablissait la perspective œedipienne. L'attention du lecteur était titillée par des histoires sordides ou tragiques, mais la narration aboutissait toujours à une conclusion conformiste et sexuellement conservatrice.

11 « For the Psyche », Time, 2 septembre 1946, p. 73-74, ici 73. 
qu'ils avaient pu manifester précédemment pour son travail (comme c'était le cas de Karl Menninger ${ }^{12}$ en particulier). Excepté Franz Alexander, ces hommes devaient devenir les acteurs principaux de la bataille, alors imminente, qui allait se livrer autour de l'harmonisation de la psychanalyse avec la religion.

En effet, le vrai visage de la psychanalyse de l'après-guerre n'était pas un émigré fraîchement arrivé aux États-Unis, mais bien Karl Menninger, de la Clinique Menninger de Topeka, au Kansas - et son frère William qui, en tant que brigadier général, avait été responsable de tous les services psychiatriques de l'armée américaine pendant la Seconde Guerre mondiale. Les frères Menninger avaient trois objectifs : contribuer à l'expansion de la psychiatrie aux États-Unis (avec une importante aide financière du gouvernement) ; rendre la psychiatrie plus psychanalytique ; contrer les attaques, provenant principalement des catholiques, qui considéraient que Freud et le christianisme étaient, en quelque sorte, incompatibles.

De fait, dans les États-Unis de l'après-guerre, la volonté de neutraliser tout ce qui pouvait ressortir d'un intérêt extrême pour la sexualité provenait in fine de la question de la compatibilité, ou de la non-compatibilité, de Freud avec le christianisme. Or, ce ne sont pas les psychanalystes qui créèrent l'étincelle qui mit le feu aux poudres en un incendie qui finirait par se répandre partout grâce aux médias généralistes et même par franchir l'océan et arriver jusqu'au Vatican. Non, c'est une histoire qui commence avec un rabbin et un évêque. En effet, Joshua Loth Liebman, un rabbin libéral de Boston, avait écrit en 1946 un livre intitulé Peace of Mind (en français : La Paix de l'esprit, traduction de Jacques Laury, Paris, Payot, 1950) qui prônait les bénéfices de l'analyse pour tous. Le livre arriva dans la liste des meilleures ventes du New York Times et y resta trois ans, y occupant même la première place pendant 58 semāines $^{-}$(non consécutives). Il visait expressément à réconcilier psychiatrie et religion. Par « psychiatrie », Liebman - en cela caractéristique de son époque entendait « psychanalyse » (de plus, il mélangeait les théories de Freud avec celles de Horney comme si cela ne posait aucun problème). Liebman, lui-même, avait été analysé, et il recommandait fortement l'analyse comme la méthode thérapeutique la plus appropriée pour obtenir une guérison, au motif qu'elle traitait les comportements destructeurs à la racine. Par « religion », Liebman entendait une foi « adulte »- en opposition à une foi «puérile » (il emploie le péjoratif childish en anglais) - en un Dieu bienfaisant et glorieux. Il s'agissait là d'un Dieu puissant, mais qui - de façon significative, et un peu à la façon de l'évangélisme social, une sorte de théologie de la libération avant la lettre - requerrait la coopération humaine pour rendre le monde

${ }^{12} \mathrm{La}$ condescendance de Zilboorg est manifeste quand il taxe Horney de naïveté pour son « refus [de reconnaître] l'intégralité des lois de la nature », pour « sa philosophie volontariste et sociologique » et son « culturalisme exclusif», de même que pour son « idéalisme anthropocentrique », qu'il estime indissociable de sa croyance dans le libre arbitre. G. ZILBOORG, "The Fundamental Conflict with Psycho-Analysis », International Journal of Psycho-Analysis, 20, 1939, p. 480-492, ici 488-489, 491 ; et G. ZILBOoRG, «The Sense of Reality », Psychoanalytic Quarterly, 10, 1941, p. 183-210, ici 209. Il existe à présent une documentation qui ne cesse de croître sur les méfaits commis par Zilboorg dans le cadre de sa pratique. 
meilleur ${ }^{13}$. Son livre rendit Liebman célèbre dans tous les États-Unis, et ses conseils pour parvenir à une meilleure acceptation de soi lui valurent des brèves et des articles de fond dans des parutions comme Life ou Ladies Home Journal (le magazine illustra l'article de nombreuses photographies de sa famille - dont une de sa fille adoptive, Leila, une adolescente polonaise, survivante d'Auschwitz. Ces photographies véhiculaient un message saisissant, à la fois témoignage de la catastrophe et message inspirant $\left.{ }^{14}\right)$. Le livre et la publicité qui l'accompagnait firent de lui-selon l'expression de l'historien Andrew Heinze - « des décennies avant Élie Wiesel [...] le premier “juif iconique" de l'Amérique d'après-guerre $»^{15}$.

Le livre provoqua également, et instantanément, la fureur de Mgr Fulton J. Sheen, son rival en célébrité médiatique cléricale. Sheen, professeur à l'Université catholique d'Amérique à Washington, était l'animateur (depuis 1930) de The Catholic Hour, une émission du soir, radiodiffusée dans tout le pays (émission qui lui valait environ 4000 lettres par semaine de ses auditeurs) et il devait bientôt devenir (en 1951) l'évêque auxiliaire de l'archevêché de New York ${ }^{16}$. Il serait, par la suite, surnommé " l'évêque américain ${ }^{17}$. En mars 1947, Sheen prononça un sermon à la cathédrale Saint-Patrick de New York où, comme le New York Times le rapporta le lendemain, il attaquait la « psychanalyse en général et la psychanalyse freudienne en particulier ». Sheen y comparait défavorablement le divan au confessionnal. Selon lui, la confession était une guérison, car elle « régénère la relation entre soi et le Père céleste ». En revanche, la psychanalyse ne pouvait pas soulager du « sentiment prégnant de culpabilité liée au péché » dont « souffrent la plupart de ceux qui consultent les psychanalystes [...]. Les victimes de la psychanalyse freudienne sont les personnes les plus détruites du monde $»^{18}$.

${ }_{13}$ Dans Peace of Mind (La Paix de l'esprit, Paris, Payot, 1950), il avance clairement que, non seulement, « loin de s'opposer, la religion et la psychiatrie se complètent mutuellement », mais aussi que "Sigmund Freud, le père de la psychanalyse, avait vraiment un objectif spirituel, même s'il n'en était peut-être pas conscient lui-même ». J. L. Liebman, Peace of Mind, New York, Simon and Schuster, 1946, p. 19 ; sur Liebman « emprunt[ant] ses concepts à la fois à Freud et à Horney », voir H. HoLt, « Liebman, Peace of Mind », American Journal of Psychoanalysis, 6, 1946, p. 51.

${ }^{14}$ D. Davidson et H. Abel, "How America Lives: Meet an American Rabbi and His Family », Ladies Home Journal, 65, janvier 1948, p. 123-31.

${ }^{15}$ Heinze peint Liebman comme celui qui, le premier, est parvenu à toucher tout le monde en devenant le symbole d'une assimilation réussie tout en proclamant la fierté de son héritage juif religieux. De plus, il a été connu comme « la première personnalité juive associée à la condition juive et à la condition humaine, après le nazisme ». Dans un pays où la notion d'héritage « judéo-chrétien » commençait tout juste à voir le jour, Liebman ne demandait pas aux juifs de s'adapter à la norme dominante chrétienne, mais, au contraire, montrait aux chrétiens ce qu'ils avaient à apprendre des juifs - aussi bien de leur tradition théologique que de Freud.

16 « Radio Religion », Time, 21 janvier 1946.

17 Th. C. Reeves, America's Bishop: The Life and Times of Fulton J. Sheen, San Francisco, Encounter Books, 2001. Voir également I. D. S. WinsBoro et M. EpPLE, « Religion, Culture, and the Cold War: Bishop Fulton J. Sheen and America's Anti-Communist Crusade of the 1950s », Historian, 71/2, 2009, p. 209-233.

18 «Sheen Denounces Psychoanalysis », The New York Times, 10 mars 1947, p. 18. 
La crise s'amplifia substantiellement quand la plus célèbre des convertis que Sheen avait réussi à ramener au catholicisme, Clare Boothe Luce - ancienne rédactrice en chef de Vanity Fair, dramaturge (à la beauté légendaire), auteure de pièces à succès à Broadway et membre du Congrès américain -, commença, en février 1947, dans le magazine féminin populaire $M c$ Call's, le récit en trois parties de sa conversion. Luce y avançait que son expérience malheureuse avec la psychanalyse - et l'obsession absurde et répugnante de cette dernière pour la sexualité - constituait « l'une des vraies raisons de sa conversion $"{ }^{19}$. Sheen poursuivit en publiant un livre intitulé Peace of Soul qui visait à contrer le Peace of Mind de Liebman. Ce livre portait un propos encore plus désagréable - car porteur d'un antisémitisme codé - sur le freudisme. Le débat envahit alors tous les médias du pays ${ }^{20}$.

On peut résumer ce qui se produisit alors de la manière suivante : les frères Menninger, William et surtout Karl, mirent au point un compromis qui fit autorité. D'une part, soucieux de s'allier, contre Horney, aux égopsychologues, qui avaient alors le vent en poupe, ils cautionnèrent la version la moins édulcorée de la théorie freudienne originelle de la libido : «De fait, la sexualité est, dans l'existence, la motivation la plus élémentaire et omniprésente, et il faut bien la comprendre pour avoir une mentalité saine ", écrivait William dans le supplément dominical du New York Times. Ils prenaient ainsi position contre les néofreudiens et leur rejet de la théorie de la libido et des pulsions. Cependant, ils affirmaient, d'autre part, que leur objectif était de réduire la polarisation de leurs patients sur la sexualité, ils promouvaient une moralité sexuelle conservatrice et avançaient que la psychanalyse était pleinement compatible avec le christianisme.

Leur hostilité à l'égard de Horney les mettait cependant en situation de blocage. Les frères Menninger voulaient proclamer (contre Horney) que, avec eux, les psychanalystes, il y était bien question de sexualité, et pas seulement d'une vague quête de sécurité dans un monde hostile ; mais, en même temps (pour contrer les attaques provenant des catholiques), ils voulaient affirmer qu'il ne s'agissait pas de sexualité - ou, du moins, pas d'une sexualité mauvaise, transgressive, mais d'une bonne sexualité affectueuse et conjugale. Karl Menninger, en particulier, entreprit une croisade pour répondre aux accusations d'immoralité. "L'actif docteur Menninger pratique le presbytérianisme autant que le freudisme ", rapportait un journaliste du Time en 1951, tandis que Menninger confiait aux lecteurs ses habitudes de prière - en

${ }^{19}$ C. B. Luce, « The 'Real' Reason », McCall's, février 1947, p. 135.

${ }^{20}$ La sexualité n'était pas le seul problème. Le libre arbitre, la culpabilité, la compatibilité de la science et de la religion : toutes ces questions étaient discutées, mais le débat revenait toujours à la sexualité, et à la théorie de la libido. Déjà, à la suite du sermon de Sheen, un journaliste du Catholic World, un mensuel très lu, indiqua expressément que, malgré les multiples révisions que Freud avait lui-même apportées, ce dernier n'avait en rien changé dans sa conviction que les problèmes individuels provenaient de l'enfance et «étaient toujours liés aux phénomènes d'excitation sexuelle et à la réaction qu'ils ont rencontrée ». " Freud renvoie toutes les impulsions à l'énergie sexuelle, sa fameuse "libido". " " Soit le freudisme a tort, ou la tradition chrétienne est absurde. » Enfin, dans The Christian Century, le journal phare du protestantisme traditionnel aux États-Unis, un luthérien s'inquiétait aussi de ce qu'il appelait, chez Freud, «l'attention démesurée pour l'énergie sexuelle ou la libido». 
termes œcuméniques. Menninger cherchait ainsi à contrer l'idée que les psychanalystes promouvaient une « philosophie sans Dieu, immorale », et il exprimait son irritation devant le fait qu' " apparemment, beaucoup de pasteurs et de laïques supposent que les freudiens sont partisans d'une liberté sexuelle généralisée ». En des termes très proches de ceux que William utilisait trois ans auparavant, mais avec encore plus de force, Karl déclarait : «Cette supposition est erronée, et la répéter, c’est colporter un mensonge, une calomnie [...]. Les psychanalystes ne sont pas partisans de la promiscuité sexuelle, ne l'encouragent pas, ne cherchent aucunement à libérer leurs patients de la honte qu'ils ressentent à son sujet [...]. Bien au contraire, la plupart d'entre eux travaillent inlassablement à libérer leurs patients de leur besoin compulsif de commettre précisément ces "immoralités" " ${ }^{21}$.

Finalement, en septembre 1952, le pape Pie XII intervint, en réaction aux arguments développés dans les médias américains, en Europe de l'Ouest et au Canada (où de nombreux prêtres et des fidèles qui partageaient un intérêt pour la psychanalyse avaient plaidé pour le droit d'être formés à l'analyse et/ou avaient publié des livres défendant la compatibilité entre Freud et la foi). En avril 1953, il céda plus encore en accordant sa bénédiction à la psychanalyse comme cure par la parole - tant que les deux interlocuteurs se gardaient de porter une attention trop grande à la sexualité. La nouvelle se répandit dans le monde entier : « Le pape Pie a officiellement reconnu l'utilisation de la psychanalyse comme un moyen de guérison. » Et : « Freud et le Vatican signent l'armistice. ") D'une certaine façon, l'évêque Sheen était alors vaincu (et cela pas seulement, bien qu'il y ait là une certaine ironie, parce qu'après avoir si vigoureusement attaqué la psychologisation du catholicisme américain, il y avait lui-même fortement contribué $^{22}$ ). Mais, d'un autre point de vue, l'évêque Sheen avait triomphé : au début des années 1950, les psychanalystes prenaient un bien plus grand soin à ne jamais communiquer au public que des idées sur la sexualité qui soient absolument normatives.

Toutefois, la sexualité, après tout, et ce, malgré les nombreuses dénégations des psychanalystes, avait été l'une des raisons principales pour lesquelles les Américains avaient eu un quelconque intérêt pour le système de pensée iconoclaste qu'était la psychanalyse. Et, hélas, l'une des conséquences malheureuses est que la psychanalyse post-néofreudienne, à laquelle les lecteurs étaient de plus en plus exposés, contribuerait tout autant, sinon davantage, à exacerber leurs problèmes en ce qui concerne la sexualité qu'à les résoudre. La réaction des psychanalystes face à une attaque inattendue provenant d'un nouveau côté n'y serait pas pour rien.

Personne, à part les porte-parole chrétiens, n'avait jusqu'alors sérieusement contesté l'hégémonie des psychanalystes sur le discours savant sur la sexualité.

${ }^{21}$ « Psychiatry and Religion », Time, 57/16, 16 avril 1951, p. 65-66.

22 Voir sur cette question : I. KRISTOL, « God and the Psychoanalysts: Can Freud and Religion Be Reconciled? », Commentary, ${ }^{\text {er }}$ novembre 1949 ; A. Nussbaum, « Profession and Faith: The National Guild of Catholic Psychiatrists, 1950-1968 », Catholic Historical Review, 93/4, octobre 2007, p. 845-865 ; C. K. GiLlesPIE S.J., Psychology and American Catholicism: From Confession to Therapy?, New York, Crossroad, 2001 ; R. Kugelmann, Psychology and Catholicism: Contested Boundaries, New York, Cambridge University Press, 2011. Sur le fait que Sheen ait fini par se radoucir, voir E. Kennedy, «Foreword», in C. K. Gillespie, Psychology and American Catholicism. From Confession to Therapy?, New York, Crossroad, 2001. 
Personne, jusqu'à ce qu'Alfred Kinsey, le sexologue, ne fasse irruption sur la scène nationale. À peine sortis de leur bataille avec l'Église, les psychanalystes s'indignèrent de l'attaque frontale contre la limitation de la sexualité à la conjugalité hétérosexuelle et monogame que représentaient les Rapports Kinsey, au seul moyen de leurs statistiques. Entre autres choses, Kinsey prouvait qu'il n'y avait pas de grande différence entre hommes et femmes, en ce qui concernait l'aptitude à atteindre l'orgasme, l'infidélité conjugale, ou encore l'intérêt pour la sexualité en général. De plus, il soutenait l'idée que l'homosexualité n'était qu'une variante naturelle de la sexualité humaine - et, de fait, une variante particulièrement répandue. Soudain, nettement moins frileux à l'idée qu'on les associe à la sexualité, les psychanalystes, poussés à la défensive par l'incroyable succès des Rapports Kinsey, se précipitèrent pour, à la fois, proclamer leur expérience de longue date en matière de sexualité et dénigrer le travail de Kinsey, en remettant frontalement en cause sa vision de la normalité de l'homosexualité, de la réalité et de la force de l'intérêt des femmes pour la sexualité. Cependant, leur mouvement tactique le plus subtil et insidieux consista à affirmer que Kinsey ne connaissait rien à l'amour. Je pourrais multiplier les exemples. En voici un, typique, de Karl Menninger : «En ce qui concerne l'orgasme considéré comme le critère principal de la sexualité, rien ne ressemble moins à un orgasme qu'un autre orgasme, tout comme il en va des baisers. » Les exemples donnés sont particulièrement significatifs : «L'orgasme d'un soldat terrifié dans la bataille, celui d'un mari amoureux dans les bras de sa femme, celui d'un homosexuel essayant désespérément de prouver sa masculinité et celui d'un monstre sadique en train de violer un enfant ne relèvent pas tous du même phénomène. » Et il poursuit en citant un autre auteur : «Il ne peut y avoir d'extase sexuelle normale que si l'élan vers l'union sexuelle procède de l'expression de l'amour pour son partenaire [...]. La promiscuité sexuelle, l'expérimentation sexuelle ou la recherche de la performance [...] dépourvues de sentiments de tendresse et d'affection sont [...] destructrices. $»^{23}$ Menninger affrontait donc Kinsey sur la place publique.

On pourrait en dire bien davantage encore sur ce que j'ai appelé la « doctrine de l'amour » et sur les dommages qu'elle a fini par causer à un nombre innombrable de personnes (on pourrait aussi montrer combien elle est éloignée de la propre conception de Freud sur la difficulté, pour beaucoup, à concilier le désir et l'amour). Mais la conclusion, ici, est la suivante : la psychanalyse freudienne aux États-Unis s'était tout d'abord, et avec une certaine habileté, transformée en réaction aux critiques fortement médiatisées des chefs de l'Église chrétienne. Les psychanalystes ne s'attendaient absolument pas à faire face à une nouvelle concurrence, mais ils sont bien parvenus à lui faire face également - et ce, pendant une bonne vingtaine d'années au moins. C'est, cependant, en s'opposant avec irritation à Kinsey qu'ils ont durci plus encore la vision du monde misogyne et homophobe pour laquelle ils sont tristement, et à juste titre, célèbres. Leurs arguments, pourtant, avaient été prédéterminés par leur querelle précédente avec l'évêque Sheen - et avant cela, avec Karen Horney, si souvent oubliée de nos jours.

${ }^{23}$ K. Menninger, « One View of the Kinsey Report », GP, 8 décembre 1953, p. 70. 


\section{Bibliographie}

« For the Psyche », Time, 2 septembre 1946, p. 73-74.

« Psychiatry and Religion », Time, 57/16, 16 avril 1951, p. 65-66.

« Radio Religion », Time, 21 janvier 1946.

«Sheen Denounces Psychoanalysis », The New York Times, 10 mars 1947, p. 18.

Anon., " Die Rolle des Juden in der Medizin », Deutsche Volksgesundheit aus Blut und Boden, août-septembre 1933, p. 15.

Aron, L. et Starr, K., A Psychotherapy for the People: Toward a Progressive Psychoanalysis, New York et Londres, Routledge, 2013.

Danto, E. A., " Have You No Shame? - American Redbaiting of Europe's Psychoanalysts », in Damousi, J. et Ben Plotkin, M. (dir.), Psychoanalysis and Politics: Histories of Psychoanalysis under Conditions of Restricted Political Freedom, New York, Oxford University Press, 2012, p. 213-231.

Davidson, D. et Abel, H., " How America Lives: Meet an American Rabbi and His Family », Ladies Home Journal, 65, janvier 1948, p. 123-131.

Gillespie, C. K., Psychology and American Catholicism: From Confession to Therapy?, New York, Crossroad, 2001.

Herberg, W., Protestant-Catholic-Jew: An Essay in American Religious Sociology, New York, Anchor Books, $2^{\mathrm{e}}$ éd, 1960. Édition française : Protestants, catholiques et israélites, la religion dans la société aux États-Unis, essai de sociologie religieuse, traduction de J. Yardley et G. Serve, Paris, Spes, 1960.

Holt, H., « Liebman, Peace of Mind », American Journal of Psychoanalysis, 6, 1946, p. 51.

Horney, K., «The Flight from Womanhood: The Masculinity-Complex in Women, as Viewed by Men and by Women ", International Journal of Psycho-Analysis, 7, 1926, p. 324-339.

Horney, K., New Ways in Psychoanalysis, New York, Norton, 1939, p. 9-10, 48, 52. Traduction française : Les Voies nouvelles de la psychanalyse, Paris, L'Arche, 1951.

Hunger, H., « Jüdische Psychoanalyse und deutsche Seelsorge », in Grundmann, W. (dir.), Germanentum, Judentum und Christentum, vol. 2, Leipzig, G. Wigand, 1943.

Kennedy, E., " Foreword ", in Gillespie, C. K., Psychology and American Catholicism. From Confession to Therapy?, New York, Crossroad, 2001.

Kristol, I., « God and the Psychoanalysts: Can Freud and Religion Be Reconciled? », Commentary, $1^{\mathrm{er}}$ novembre 1949.

Kruse, K., One Nation Under God: How Corporate America Invented Christian America, New York, Basic Books, 2015.

Kugelmann, R., Psychology and Catholicism: Contested Boundaries, New York, Cambridge University Press, 2011.

KurIloff, E., " Revelations in Psychoanalytic History », Psychoanalytic History, Contemporary Psychoanalysis, 45/4, 2009, p. 577-580.

Liebman, L., Peace of Mind, New York, Simon and Schuster, 1946.

LuCE, C. B., « The 'Real' Reason », McCall's, février 1947, p. 135. 
McLaughlin, N., « Why Do Schools of Thought Fail? Neo- Freudianism as a Case Study in the Sociology of Knowledge », Journal of the History of the Behavioral Sciences, 34/2, printemps 1998, p. 113-134.

Menninger, K., « One View of the Kinsey Report », GP, 8 décembre 1953, p. 70.

Nussbaum, A., « Profession and Faith: The National Guild of Catholic Psychiatrists,

1950-1968 », Catholic Historical Review, 93/4, octobre 2007, p. 845-865.

Peale, N. V., The Power of Positive Thinking, New York, Prentice-Hall, 1952.

Première édition française : Votre pensée peut tout, traduction de S. Lechevrel, Paris, Les Presses de la Cité, 1955.

Reeves, Th. C., America's Bishop: The Life and Times of Fulton J. Sheen, San Francisco, Encounter Books, 2001.

Winsboro, I. D. S. et Epple, M., « Religion, Culture, and the Cold War: Bishop Fulton

J. Sheen and America's Anti-Communist Crusade of the 1950s », Historian, 71/2, 2009, p. 209-233.

ZILboorg, G., « The Fundamental Conflict with Psycho-Analysis », International Journal of Psycho-Analysis, 20, 1939, p. 480-492.

ZILboorg, G., « The Sense of Reality », Psychoanalytic Quarterly, 10, 1941, p. 183-210. 